\title{
How Differences in the Disease Process of the COVID-19 Pandemic Pose Challenges to the Delivery of Critical Care Nutrition
}

\author{
Mohamed Eisa $^{1}\left[\right.$ - Stephen A. McClave ${ }^{1} \cdot$ Sally Suliman ${ }^{2} \cdot$ Paul Wischmeyer $^{3}$ \\ Accepted: 24 September 2021 / Published online: 21 October 2021 \\ ( ) The Author(s), under exclusive licence to Springer Science+Business Media, LLC, part of Springer Nature 2021
}

\begin{abstract}
Purpose of Review The COVID-19 pandemic is a unique disease process that has caused unprecedented challenges for intensive care specialists. The hyperinflammatory hypermetabolic nature of the disease and the complexity of its management create barriers to the delivery of nutritional therapy. This review identifies the key differences which characterize this pandemic from other disease processes in critical illness and discusses alternative strategies to enhance success of nutritional support. Recent Findings Prolonged hyperinflammation, unlike any previously described pattern of response to injury, causes metabolic perturbations and deterioration of nutritional status. High ventilatory demands, hypercoagulation with the risk of bowel ischemia, and threat of aspiration in patients with little or no pulmonary reserve, thwart initial efforts to provide early enteral nutrition (EN). The obesity paradox is invalidated, tolerance of EN is limited, intensivists are reluctant to add supplemental parenteral nutrition $(\mathrm{PN})$, and efforts to give sufficient nutritional therapy remain a low priority.

Summary The nature of the disease and difficulties providing traditional critical care nutrition lead to dramatic deterioration of nutritional status. Institutions should not rely on insufficient gastric feeding alone but focus instead on redoubling efforts to provide postpyloric deep duodenal/jejunal EN or re-examine the role of supplemental PN in this population of patients with such severe critical illness.
\end{abstract}

Keywords COVID-19 disease $\cdot$ Coronavirus $\cdot$ SARS-CoV-2 $\cdot$ Nutritional therapy $\cdot$ Enteral nutrition $\cdot$ Parenteral nutrition · Malnutrition

\section{Introduction}

Since the early part of 2020, the emergence of the COVID19 pandemic has posed incredible personal and professional challenges for healthcare providers charged with treating this complex group of patients. While we have learned much

This article is part of the Topical Collection on Gastroenterology, Critical Care, and Lifestyle Medicine

Mohamed Eisa

mohamed.eisa@louisville.edu

1 Division of Gastroenterology, Hepatology, and Nutrition, University of Louisville School of Medicine, 550 S. Jackson St, Louisville, KY 40202, USA

2 Division of Pulmonary, Critical Care \& Sleep Disorders Medicine, Louisville, USA

3 Division of Anesthesiology and Critical Care Medicine, Duke University Hospital, Durham, North Carolina, USA about the disease process leading to many improvements in its clinical management, unfortunately, efforts to provide adequate nutritional therapy have not been nearly as successful.

At the onset of the pandemic, any strategies adopted for providing nutritional therapy to this new patient population was based on guidelines previously published for providing traditional nutritional therapy to critically ill patients in an intensive care unit (ICU) $[1 \bullet, 2 \cdot]$. However, it quickly became evident that the wide range of symptoms, the sheer volume of patients, and the unpredictability of the disease process combined with excessive demands for oxygenation created barriers limiting the successful delivery of such nutritional therapy. The objectives of this manuscript are to highlight the unique nutritional and metabolic features of the critically ill patient with COVID-19 and understand the role that obesity plays in this disease process. Also, it is important to understand how the constraints of COVID19 disease impact the delivery of early enteral nutrition 
(EN), and to learn appropriate changes in strategy to provide nutritional therapy despite considerable barriers in a manner that is appropriate for each individual institution or medical center.

\section{Key Difference: The Nature of the Pandemic}

The SARS-CoV-2 virus that causes COVID-19 disease shares a unique subclass with the viruses associated with the severe acute respiratory syndrome (SARS) and the Middle Eastern respiratory syndrome (MERS), which were responsible for two pandemics that preceded the current COVID-19 pandemic (Fig. 1) [3, 4]. SARS started in Hong Kong in 2003, spreading through Asia, causing 8098 people to be infected and resulting in a $9.7 \%$ mortality [5]. Ten years later in 2012 after the SARS pandemic, the MERS pandemic started in Saudi Arabia and spread as far as Korea. A total of 2494 patients were infected and had a $34 \%$ mortality rate. In comparison, the SARS-CoV-2 virus causing the COVID-19 pandemic started in 2019 and over the first 18 months resulted in 116 million individuals worldwide being infected. COVID-19 disease initially had a mortality rate of $4.4 \%$, but after 18 months with improvements in management, the cumulative mortality rate was reduced to a rate of $2.2 \%$ [5]. The original hosts for the SARS-CoV-2 virus were bats, but infestation quickly spread to an intermediate host (the Chinese Pangolin or ant-eater), and then to humans. This is one example of a virus jumping from one animal species to another (Fig. 1) [3, 4].

COVID-19 is a uniquedisease process, which creates its own set of complications. First, a full spectrumof disease phenotypes exists, ranging from completely asymptomatic individuals tothose who experience severe critical illness in the form of acute respiratorydistress syndrome (ARDS), often requiring multiorgan support in an intensivecare setting. Fortunately, $80 \%$ of those patients infected have mild disease, $15 \%$ have symptoms that require hospitalization, and only $5 \%$ require intensivecare. [6, 7] At thestart of the pandemic, $75 \%$ of the ICU patients were requiring mechanicalventilation, a percentage that dropped significantly to $25 \%$ as strategies formeeting ventilatory demands were adopted to prevent intubation.

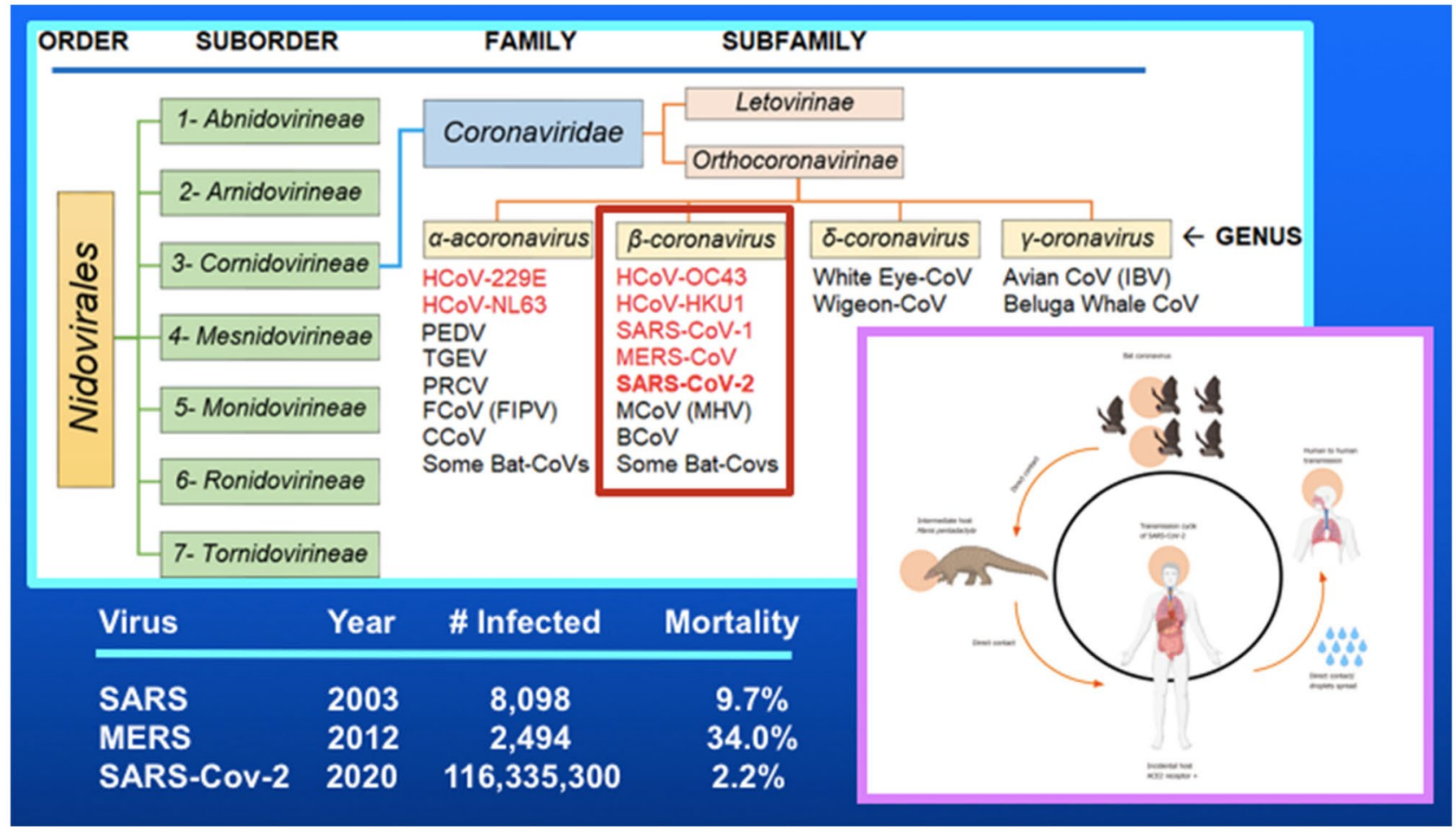

\section{Legend: SARS = Severe Acute Respiratory Syndrome; MERS = Middle Eastern Respiratory Syndrome; SARS-CoV-2 = Severe Acute Respiratory Syndrome Coronavirus-2.}

Fig. 1 Classification of SARS-CoV-2 Virus (Figure adapted with permission from $\mathrm{H}$ A. Aboubakr et al. and Galanopoulos $\mathrm{M}$ et al. references [3, 4]). Legend: SARS severe acute respiratory syndrome,
MERS Middle Eastern respiratory syndrome, SARS-CoV-2 severe acute respiratory syndrome coronavirus-2 
For a variety of reasons, the process of making recommendations for the nutritional therapy of those COVID-19 patients with severe critical illness was not easy early in the pandemic, and factors which impacted those recommendations did not remain consistent as the pandemic progressed. Quickly it became apparent that the disease process violated many of the existing standards of traditional critical care nutrition. The position on the pandemic curve of new cases for a particular region or location could have profound implications in terms of supplies (personal protective equipment or PPEs) and medications. Additionally, institutional culture as well as the leadership and expertise in a given ICU had tremendous influence on local protocols and what patients would actually receive in terms of supplies, nutrients, and supplemental therapies. In order to improve the delivery of nutrition in this new pandemic, intensivists would need to reassess their biases and existing prejudices with regard to traditional critical care nutrition [8].

\section{Key Difference: Lack of Effective Guidelines for Nutritional Therapy}

At the beginning of the pandemic, there was an urgent need for the Society of Critical Care Medicine (SCCM) and the American Society for Parenteral and Enteral Nutrition (ASPEN) to provide clinicians with guidelines to manage this new population of critically ill patients. A group of experts was gathered to provide guidelines for nutritional therapy, which were developed in March 2020, placed on the SCCM website, and eventually published in the Journal for Parenteral and Enteral Nutrition [1•]. Given the lack of data on COVID-19 patients at the time, basic principles for nutritional therapy were adopted from traditional critical care delineated in the ASPEN/SCCM 2016 guidelines [2•]. Those basic principles were centered on early gastric feeding with a gradual increase in the infusion rate to goal. The emphasis on early EN included those patients who required prone positioning, extracorporeal membrane oxygenation (ECMO), or paralysis by neuromuscular blockade. Also, the early nutritional guidelines emphasized the use of exclusive parental nutrition (PN) as soon as possible if EN was not feasible. There was no mention of adding supplemental PN to EN when the amount of tube feeding was insufficient [1•].

A national survey was conducted by the University of Louisville from September 2020 to December 2020 in order to expand collective knowledge on the status of providing nutritional therapy for this difficult group of patients. The survey was sent out by email, social media, and newsletter to members of ASPEN. Of the 440 individuals who viewed the link, $45 \%$ responded by completing the survey, of which $30 \%$ were physicians and $70 \%$ were dietitians. Responses were equally split between healthcare providers at community (51\%) and academic medical centers (43\%). At the time of this survey, $49 \%$ of the centers had a protocol to treat COVID-19 patients, but only $21 \%$ had a protocol for providing their nutritional therapy. Some of the key issues raised by their responses included the difficulty in feeding a patient undergoing awake proning and the reluctance to add supplemental PN to insufficient EN. Results of the survey indicated that an emphasis solely on gastric feeding was rarely effective in this group of patients [9•].

\section{Key Difference: Dynamic Changes in Overall Management}

Throughout the pandemic, clinicians were forced to deal with a rapidly evolving dynamic process where information was being accumulated around the globe and researchers were scrambling to provide objective evidence by which to manage these patients. Such an environment made it difficult to standardize or protocolize treatment, and to quickly apply the academic experience to community centers. As would be expected, there were many strategies that worked, but several others which failed to be effective. For instance, the availability of several therapeutic agents such as convalescent serum and hydroxychloroquine early on appeared promising but were later found to have little effect on outcome. In contrast, the antiviral agent Remedesivir, the production of monoclonal antibodies (Baricitinib, Tocilizumab), and eventually the development of mRNA vaccines appeared particularly effective and may have contributed successfully to the reduction in mortality. Early in the pandemic, melatonin was recommended for use in COVID-19 disease with several micronutrients such as vitamin D, vitamin C, zinc, and selenium because each agent demonstrated antiviral properties, antioxidant effects, and had been shown to reduce systemic inflammation. While the micronutrients so far have not been shown to change outcome appreciably and thus have not gained generalized utilization, use of melatonin has continued because of its additional anti-psychotic effects and benefit in promoting sleep in these patients who are so often sleep-deprived and vulnerable to ICU-induced psychosis.

There was a major shift in the strategies to meet oxygenation demands and the management of the hyperinflammatory response in these patients. At the beginning of the pandemic, the recommended strategy was early invasive mechanical ventilation (IMV). However, as more data emerged, there was a shift towards alternative treatment strategies such as use of non-invasive positive pressure ventilation (NIPPV) and high flow oxygen modalities. Clinicians were advised 
Fig. 2 The Obesity Paradox (figure reproduced with permission from D L. Davenport et al. - reference [14])

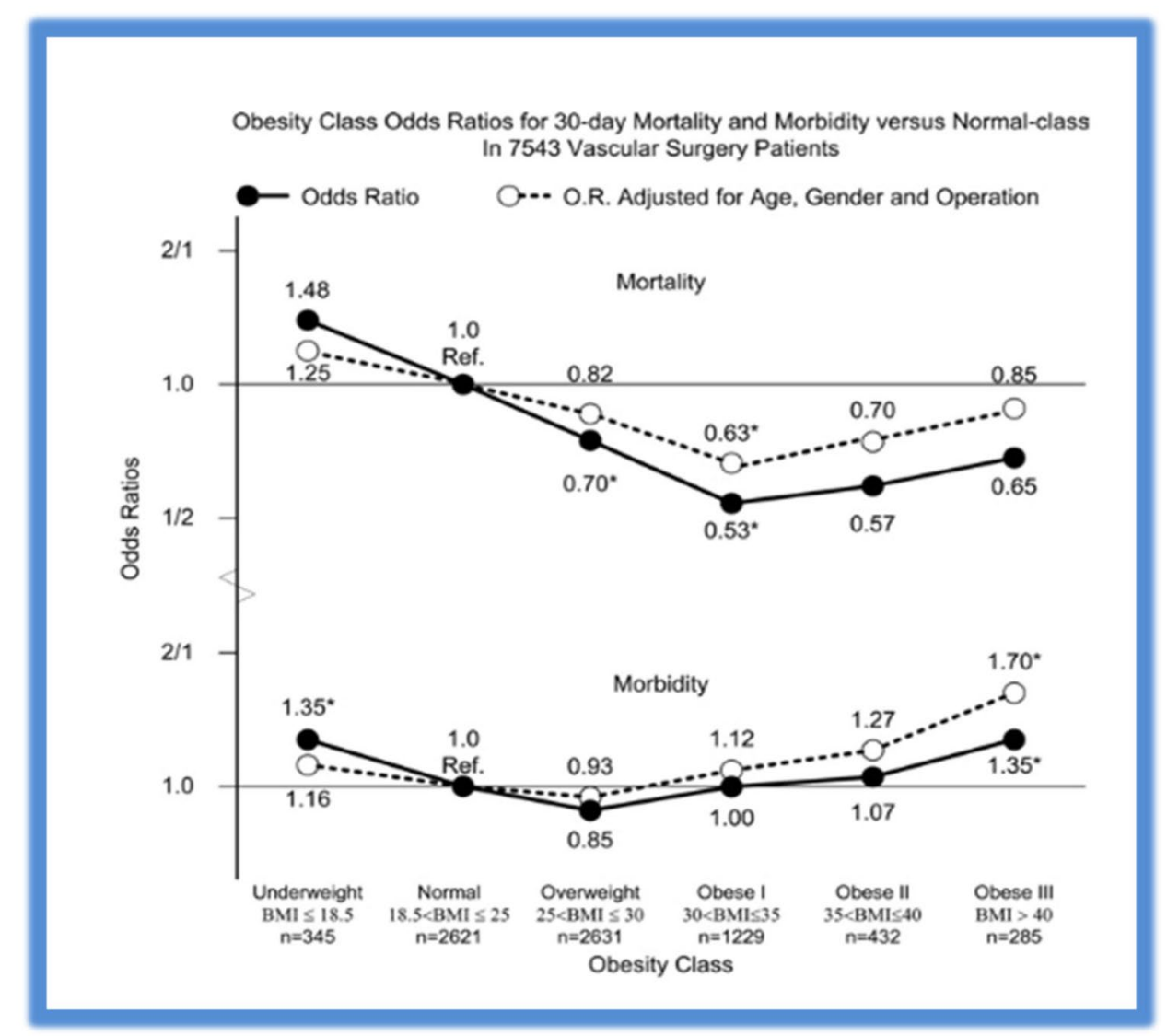

against using steroids early on, but outcome was shown to be better with aggressive early use of corticosteroids. Despite generalized fatigue of healthcare workers and the politicization of public health measures, these rapidly evolving measures resulted in better more successful treatment of COVID-19 disease as evidenced by a reduction in mortality from 4.5 to $2.2 \%$ [10].

\section{Key Difference: Invalidation of the Obesity Paradox}

For some time, observational data describing the obesity paradox have provided reassurance to intensivists with the implication that patients who are overweight or have class I or class II obesity have better outcome with reduced mortality compared to patients with the normal body mass index (Fig. 2) [11-14]. Experience with the pandemic so far appears to invalidate the obesity paradox, at least for the COVID-19 disease process. Obesity increases the likelihood of contracting COVID-19 disease, and is a risk factor for greater disease severity, poorer outcome, and increased mortality [13, 14]. It increases the risk for development of ARDS and severe pneumonia [15, 16]. The presence of obesity is ranked second after age (over 60 years) as a risk factor for serious and severe disease [10]. Obesity increases the likelihood of hospital admission by twofold compared with non-obese patients with COVID-19 [11]. Other factors which contribute to greater disease severity and mortality include cardiovascular disease, diabetes, chronic respiratory disease, hypertension, malignancy, lower socioeconomic status, male sex, and pre-existing malnutrition. [11-13].

A potential reason for higher mortality in obese patients with COVID-19 is that ACE-2 receptors may be expressed at the highest level in adipose tissue and the lungs. Also, obese patients may have other comorbidities such as obstructive sleep apnea (OSA) which causes baseline inflammation and cardiac strain such as pulmonary hypertension.

Clinicians have difficulty determining caloric requirements in the obese patient with COVID-19 disease, as weight-based predictive equations are less accurate and indirect calorimetry is impractical given the necessary infectious precautions. These factors combined with poor delivery of the nutritional regimen result in significant weight loss throughout their hospitalization, which raises questions about the appropriateness of the high protein low calorie recommendations. Such a strategy needs to be reevaluated in this disease process [15-17]. 
Fig. 3 Prioritization of responsibilities in the complex management of the critically ill patient with COVID-19 disease. Legend: PPE personalized protective equipment, $\mathrm{Rx}$ therapy

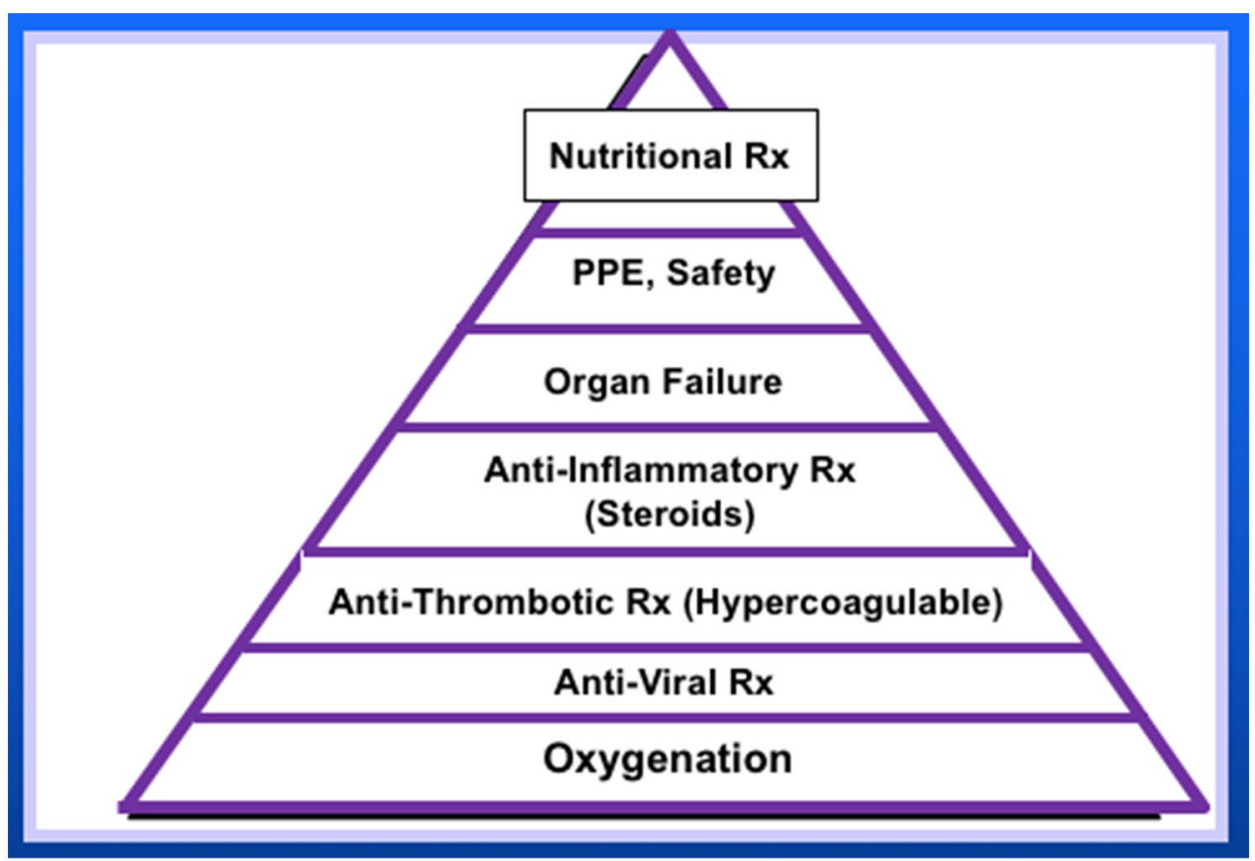

Legend: $\mathrm{PPE}=$ Personalized Protective Equipment; $\mathrm{Rx}=$ Therapy.

\section{Key Difference: Imposing List of Management Priorities}

Traditionally, provision of nutritional therapy can be a low priority in the management of critically ill patients. This tendency is amplified by the challenges and the sheer number of higher priority items in the complex management of the patient with COVID-19 disease (Fig. 3). On arrival to the ICU, these patients are generally profoundly hypoxemic, and so their ventilatory demands take the highest priority in the initial management. The next three pillars of therapy are anti-viral therapy, anti-thrombotic therapy given their hypercoagulation, and steroids to treat the hyperinflammatory state. Subsequently, attention is often shifted towards the management of multiple organ failure, use and preservation of personalized protective equipment (PPE), and assuring staff safety. Once all these management strategies are optimized, then attention can be turned to the delivery of nutritional therapy, considered unfortunately as one of the lowest priorities. As nutritional therapy is initiated, clinicians find that their patients have loss of taste and smell, and experience poor appetite, all of which is made worse by aerophagia while on NIPPV. Intensivists are reluctant to add another procedure just for placement of a feeding tube, especially one with the potential risk for aerosolization. Other concerns among providers caring for these patients on NIPVV is the fear of aspiration and the potential for a break on the seal of the bi-level positive airway pressure (BIPAP) mask that the feeding tube can cause. Any thought of adding supplemental PN to insufficient tube feeding is dampened by concern for worsening hypertriglyceridemia and hyperglycemia. These barriers to the delivery of nutritional therapy and its overall position in the imposing list of priorities lead to an end result of weight loss and increasing risk of refeeding syndrome throughout hospitalization in the ICU (Fig. 3).

\section{Key Difference: Unique Metabolic Response}

The metabolic response to COVID-19 is unique, differing from every other model of stress response to injury that has been described previously in the past (Fig. 4a-d). Using serial measurements by indirect calorimetry, Wischmeyer has shown a pattern of prolonged hypermetabolic energy expenditure, essentially extending the acute and immediate post-acute phases of critical illness out 3 to 5 weeks [18•]. In this report, energy expenditure was shown to remain close to that which would be expected for normal resting energy expenditure (REE), at $95 \%$ of that predicted by the Harris Benedict equation, during the first week. After that point, the patients become hypermetabolic, averaging 124\% the Harris Benedict predicted value in the second week and even higher at $143 \%$ for the third week of hospitalization in the ICU (Fig. 4a) [18•]. The late prolonged peak of this model is different from the Cuthbertson model of Ebb and Flow phase of injury (Fig. 4b) [19], the Systemic Inflammatory Response Syndrome (SIRS)/Compensatory Antiinflammatory Response Syndrome (CARS) model (Fig. 4c) 
Fig. 4 Comparison of the metabolic response to injury in COVID-19 disease (a) (figure adapted with permission from J Whittle et al. - reference 18) to previously described models: Cuthbertson Ebb/Flow (b) (figure reproduced with permission from JS Carson et al. - reference [19]). SIRS/CARS (c) and PICS (d) (figures reproduced from B Mathias et al. - reference [20]). Legend: SIRS systemic inflammatory response syndrome, CARS compensatory anti-inflammatory response syndrome, PICS persistent inflammatory catabolism syndrome

[20], and the Persistent Inflammation Catabolism Syndrome (PICS) (Fig. 4d) [20]. All three of these later models peak within 5 to 7 days before returning to normal baseline (Ebb/ Flow), being offset by the opposing hypometabolism (SIRS/ CARS) or failing to return completely to baseline over several weeks of hospitalization (PICS).

The hyperinflammatory response during COVID-19 illness is a systemic phenomenon that leads to cytokine storm with elevation of systemic markers, such as C-reactive protein (CRP) and erythrocyte sedimentation rate (ESR). Evidence that this systemic response is extended to individual organ systems is shown by elevation of myoglobin (muscle), troponin (heart), and aspartate transaminase/alanine transaminase (liver). The extended period of hyperinflammation causes several metabolic derangements such as severe insulin resistance, volume overload, and hypernatremia secondary to insensible loss and osmotic diuresis. Patients typically get hypocalcemia, hyperkalemia or hypokalemia, hyperphosphatemia (secondary to muscle breakdown and mitochondrial failure), and hypertriglyceridemia [21, 22]. Due to the difficulties in providing nutritional therapy for these patients, their risk of refeeding syndrome may actually increase throughout their ICU stay. The net result of these metabolic derangements is severe deconditioning, immobilization, and accelerated proteolysis of muscle mass.

\section{Key Difference: Excessive Ventilatory Demands}

The excessive ventilatory demands and the efforts needed to meet those demands may be the most prominent factor impeding delivery of nutritional therapy. In traditional critical care, use of early EN has been shown to reduce pneumonia and other infectious morbidity. In COVID-19 disease, however, the threat of aspiration from $\mathrm{EN}$ in a patient with little or no pulmonary reserve thwarts more aggressive efforts at providing nutritional support.

With the need to prevent intubation and placement on IMV, the use of proning has been increasingly emphasized in the management of the patient with COVID-19. Proning has been shown to improve ventilation, promote clearance of secretions, and reduce mortality [23]. The University of Louisville survey found that $18 \%$ of patients could sustain awake voluntary proning for only 4-6 h, 30\% sustained for

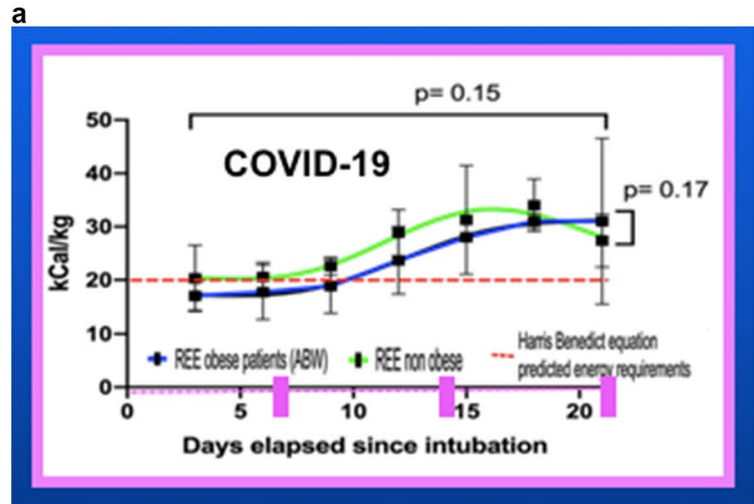

b

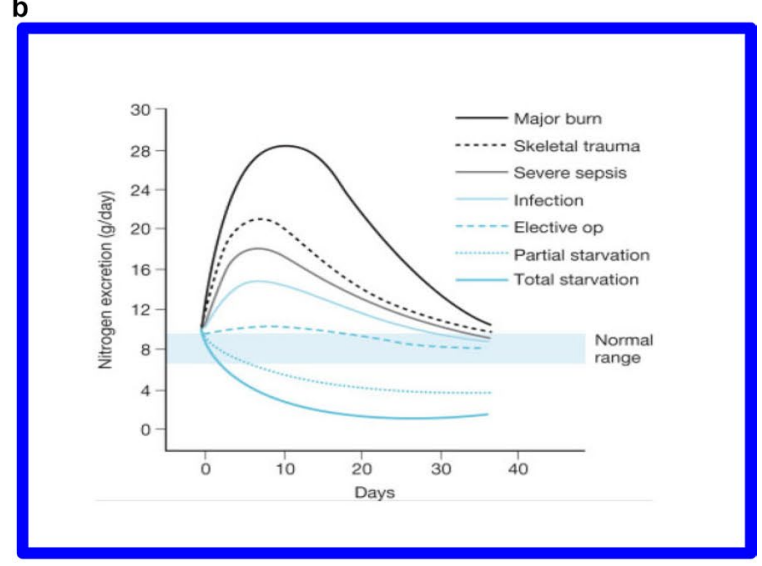

C

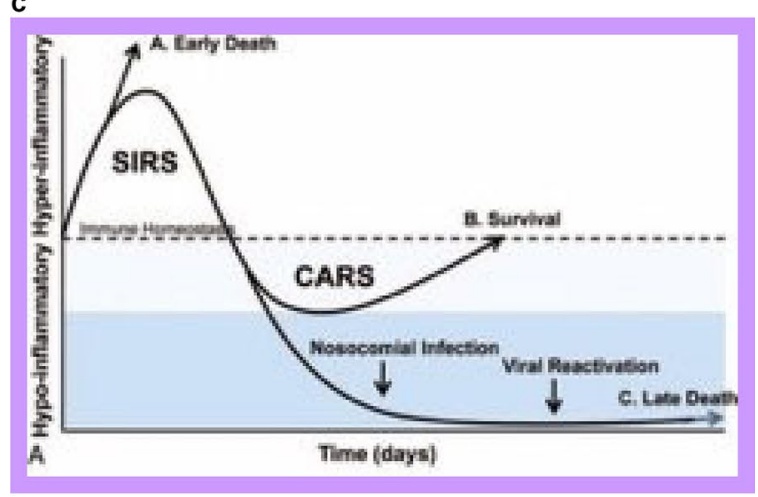

d

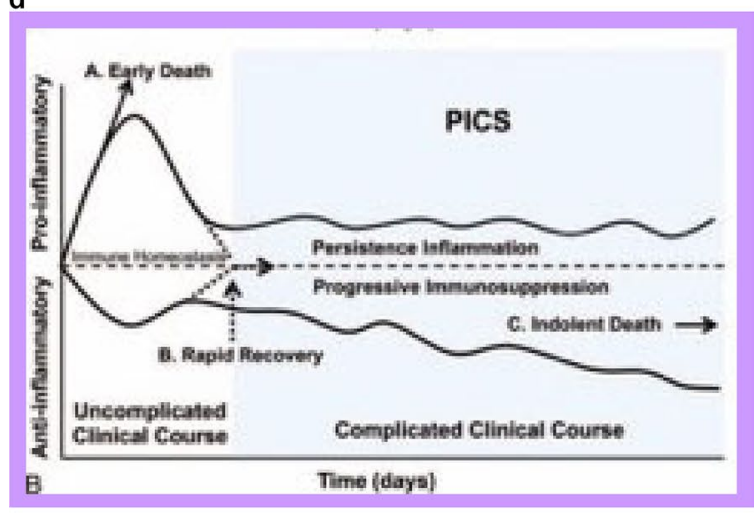

Legend: SIRS = Systemic Inflammatory Response Syndrome; CARS = Compensatory AntiInflammatory Response Syndrome; PICS = Persistent Inflammatory Catabolism Syndrome. 
more than $6 \mathrm{~h}$, but the majority of patients (44\%) were simply asked to attempt proning "as tolerated." The nutritional therapy recommended for patients during awake voluntary pronating involves oral intake as tolerated in terms of small frequent meals or oral supplements through a straw. Feedings are timed with rotation, such that the last intake occurs $1 \mathrm{~h}$ before patients are placed in the prone position to reduce risk of aspiration. If patients fail to meet their requirements with oral intake, a nasogastric feeding tube is recommended after 5-7 days [9].

Once patients are intubated, sedated, and placed on mechanical ventilation, longer periods of proning can be utilized. The University of Louisville survey indicated that $13 \%$ sustained proning for $7-12 \mathrm{~h}$, while $58 \%$ tolerated up to 13-18 h. [9] The SCCM website guidelines for feeding in this situation recommended intragastric EN along with elevation of the head of the bed to $25^{\circ}$ to reduce the risk of aspiration, facial edema, and intraabdominal hypertension. Given how cumbersome shifting of patients can be from the supine to the prone position and vice versa, tube feeding should be discontinued immediately before shifting to avoid accidental dislodgment [24]. Typically, in practice, tube feeding is markedly reduced while proning such that patients arbitrarily receive only $25 \%$ of their goal feeding while prone, increasing to $100 \%$ of goal when they are placed back supine. Previous studies done prior to the COVID-19 pandemic would suggest that this practice is not necessary. A study by Reignier showed that use of a proning protocol, which consisted of a prophylactic prokinetic and elevation of the head of bed to $25^{\circ}$, resulted in an increase in tidal volume (TV) and greater rate of delivery of EN, with no increase in nausea, vomiting or ventilator-associated pneumonia (VAP) [25]. Another study by Sams showed no difference in aspiration while proning between gastric and postpyloric feeding [26]. Yet Reignier showed that providers were twice as likely to stop EN when non-COVID patients were in prone (82\%) vs supine (49\%) position, indicating a pre-existing bias that has most likely been carried over to the pandemic [27].

NIPPV with a BiPAP mask is typically used beforehand to prevent the need for intubation and placement on IMV, or afterward as a step-down measure $24-48 \mathrm{~h}$ post-extubation. Some providers believe NIPPV is a relative contraindication to EN, given the difficulty in maintaining a seal on the mask with a nasogastric tube in place. Subsequently, many of these patients are kept NPO [1•]. The University of Louisville survey found that $39 \%$ of patients were kept nil per os (NPO), $28 \%$ received gastric feeding, $17 \%$ received postpyloric feeding, and only $5 \%$ received PN [9].

Extra-corporeal membrane oxygenation (ECMO) is used most often as a last resort after failure of mechanical ventilation. There is a perception of increased risk of gastric feeding because of delayed gastric emptying and risk of ischemic bowel. However, previous reports suggest that early use of EN for patients on ECMO has a better outcome with decreased mortality than delaying the initiation of EN [28, 29]. With the use of paralytics and neuromuscular blockade, Ohbe showed that early vs delayed EN resulted in decreased hospital mortality and hospital length of stay (findings that may be explained by the fact that neuromuscular blockade affects only skeletal muscle and not the smooth muscle of the GI tract) [30].

\section{Key Difference: Reluctance to Utilize Supplemental PN}

The role of supplemental PN added to insufficient EN in traditional critical care has been limited in the past. Greater use of this strategy may be required in the COVID-19 pandemic due to the troublesome barriers in delivering EN. The ASPEN/SCCM 2016 guidelines stated that in traditional critical care, practitioners should consider adding supplemental PN only after 7-10 days if EN alone is insufficient $(<60 \%$ goal) regardless of the risk [2•]. The key issue with COVID-19 patients is that they often sustain prolonged inadequate nutritional support, and their disease course is not always predictable. Throughout the COVID-19 pandemic, there appeared to be a reluctance among critical care providers to add supplemental PN. In the past, PN was considered harmful and even described as "poisonous" when utilized in critical illness compared to use of EN [31]. For the past decade, intensivists have believed that trophic feeding or permissive underfeeding should be sufficient for critically ill patients with similar outcomes to full feeding [32]. During the COVID-19 pandemic, specifically, the requirement for so many intravenous medications and their infusion pumps resulted in the intensivists being reluctant to dedicate central access to the use of PN. Additional concern centered on the premise that use of supplemental PN would worsen glycemic control and hypertriglyceridemia.

But the strongest argument for failing to add supplemental PN to insufficient EN in critically ill patients with COVID-19 disease was the lack of evidence to date in the literature that such practice would have any benefit on patient outcomes (Table 1). Two trials were designed simply to evaluate the concept of supplemental PN. The EPaNIC trial showed that early vs late supplemental PN was associated with worsening morbidity including increased risk of infections and hospital length of stay [33]. The Heidegger Supplemental Parenteral Nutrition (SPN) trial showed reduced risk of nosocomial infections with supplemental PN; however, those infections cited were considered minor infections (skin, nose, etc.) and there was no difference in major infections such as pneumonia or bacteremia [34 ]. One trial was designed to evaluate "goal-directed therapy" 
Table 1 Impact of supplemental parenteral nutrition on clinical outcomes

\section{Full feeding group Underfeeding com- Outcomes \\ parison group}

\begin{tabular}{|c|c|c|c|}
\hline \multicolumn{4}{|l|}{ Supplemental PN } \\
\hline EPaNIC Trial 2011 & Early $(100 \%$ day 7$)$ & Late (45\% day 7 ) & $\uparrow$ Infect, LOS, $\downarrow$ dschg alive \\
\hline Heidegger Trial 2013 & SPN $(103 \%)^{a}$ & EN $(77 \%)$ & $\downarrow "$ Other" infections \\
\hline \multicolumn{4}{|l|}{ Goal-Directed } \\
\hline EAT-ICU Trial 2017 & Goal $(90.7 \%)^{\text {a }}$ & Std (56.2\%) & No difference \\
\hline \multicolumn{4}{|l|}{ Tight Calorie Control } \\
\hline Pilot TICACOS 2011 & Tight $(105.5 \%)^{\text {a }}$ & Liberal (80.5\%) & $\uparrow \mathrm{MV}$, ICU/Hosp LOS \\
\hline Internat TICACOS 2020 & Tight $(89.4 \%)^{\text {a }}$ & Liberal (66.9\%) & No difference \\
\hline TOP-UP Trial 2017 & Top-Up (95\%) ${ }^{\text {b }}$ & EN (69\%) & No difference \\
\hline
\end{tabular}

${ }^{\mathrm{a}}$ Used indirect calorimetry

${ }^{\mathrm{b}}$ Weight-based equation where requirements were measured by indirect calorimetry and supplemental PN was added to insufficient EN to meet those requirements. Results of this EAT-ICU trial showed no difference between the study group meeting therapeutic goals compared to controls on EN alone [35]. Three other trials reported on the value of supplemental PN through the lens of "tight calorie control," where specific requirements were determined and supplemental PN similarly was added to EN to meet those requirements. The single center "pilot" TICACOS trial showed worse outcomes in the group receiving supplemental PN with an increase in duration of mechanical ventilation and hospital LOS, albeit a trend towards reduced mortality was seen compared to controls on "liberal" EN alone [36]. The other two trials, the "international" multicenter TICACOS trial and the TOP-UP trial [37, 38], showed no difference in outcomes between study patients and controls. While these trials were done in non-COVID-19 patients, experience with the pandemic has shown that the patients with COVID-19 disease are different with a sustained hypermetabolic state and a prolonged increase in energy and protein requirements. As such, one might argue that any reluctance to add supplemental PN to continued inadequate EN should be reconsidered, as the alternative (which is the current practice of EN alone) appears to perpetuate deterioration of nutritional status.

\section{Key Difference: Perceived Need for Micronutrient Supplementation}

The use of empiric micronutrient supplementation is controversial in traditional critical care. The increasing oxidative stress of critical illness can deplete micronutrients involved in antioxidant defense even in patients whose levels were replete prior to injury (Fig. 5). [39] Outcome benefits are variable, doses have yet to be standardized, little emphasis is placed on documenting deficiencies prior to

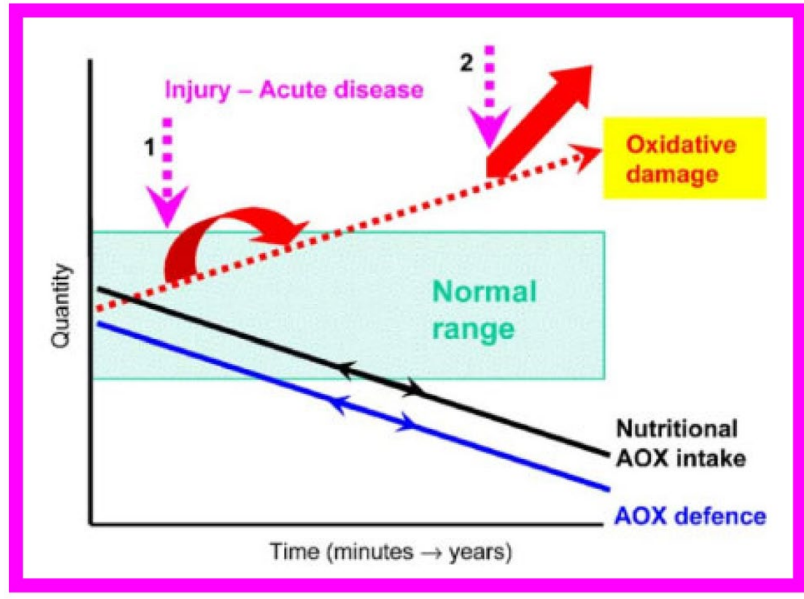

Legend: AOX $=$ Antioxidant .

Fig. 5 Pattern of antioxidant micronutrients in response to metabolic stress (figure reproduced with permission from MM Berger - reference [39]). Legend: AOX antioxidant

supplementation, and recommendations from European and North American nutrition societies are discordant. Renewed interest on use of micronutrient supplementation arose early in the COVID-19 pandemic. Four specific micronutrients (zinc, selenium, vitamin D, and vitamin C) have been shown to have antioxidant, anti-inflammatory, and antiviral properties, which garnered attention for their potential role in preventing, treating, or at least attenuating the disease process of COVID-19. While little data in the form of randomized controlled trials (RCTs) are available and formal recommendations through societal guidelines are not yet feasible, nonrandomized observational trials suggest potential benefit from the provision of these four micronutrients.

Twenty-two RCTs have evaluated the use of Selenium vs placebo or other antioxidant micronutrients in nonCOVID-19 critically ill patients. A systematic review of 
this data showed no difference in mortality or nosocomial infections [40]. Two observational trials, however, have been published evaluating the use of selenium in ICU patients with COVID-19 disease. One trial measured the content of selenium in patients' hair, and showed that higher content at the onset of disease was associated with a higher cure rate [41]. A second trial examined blood levels of selenium in survivors vs non-survivors in COVID-19, and showed that those patients discharged home had higher levels of selenium than those who died [42]. While the findings of these observational studies are provocative, the data are not sufficient to formally recommend therapy.

A meta-analysis of zinc supplementation vs placebo in four small RCTs in non-COVID-19 patients with burns and trauma showed no benefit from this micronutrient in any of the outcome parameters measured (mortality, infections, length of stay, or symptoms) [43]. In COVID-19 patients, a prospective non-randomized cohort multicenter study of zinc supplementation showed an increased rate of being discharged home and a significant reduced risk of in-hospital mortality compared to placebo. The zinc used in the study was combined with a zinc transporter, which was hydroxychloroquine [44]. In a separate study, the COVID19 A-to-Z Multicenter RCT compared ambulatory patients who received either zinc, vitamin $\mathrm{C}$, or both, with an endpoint of a 50\% reduction in symptoms. Results showed no benefit of either supplement in comparison to standard of care [45].

A plethora of data exists on the use of vitamin $\mathrm{D}$ in non-COVID ICU patients. One meta-analysis showed benefit from the use of vitamin $\mathrm{D}$, while another metaanalysis showed no benefit $[46,47]$. The PETAL VIOLET double-blind RCT first documented low vitamin D levels, after which study patients received a single enteral dose of 500,000 IU which subsequently was documented to normalize levels. However, results showed no mortality benefit from use of vitamin D [48]. In COVID-19 patients, there have been seven observational trials, five of which showed a correlation between vitamin D levels and improved outcome (the higher the level, the better the outcome) [49-55]. The PO Calciferol RCT evaluated hospitalized COVID-19 patients to see whether receipt of oral vitamin D vs placebo would prevent subsequent transfer to the ICU. Results showed that $98 \%$ of those who received vitamin D did not require ICU admission vs 50\% who received placebo [56]. Currently, the large VITDALIZE trial is still in phase III and 41 trials are currently registered in clinicaltrials.gov looking at use of vitamin D in COVID-19 patients.

A large body of data exists on use of vitamin $\mathrm{C}$ in nonCOVID-19 patients, mainly because of the interest in HAT therapy (Hydrocortisone, Ascorbic Acid, and Thiamine) in critical illness. There are seven RCTs, three of which showed minor benefits in terms of decreased hours of vasopressor therapy, faster clearance of lactic acidosis, and more rapid improvement in Sequential Organ Failure Assessment (SOFA) score [57-63]. On the other hand, the large CITRIS-ALI RCT in non-COVID patients showed better 28-day mortality, increased ICU-free days, and increased transfer out of the ICU by day 6 in patients who received vitamin C vs placebo [64]. However, the authors indicated the study did not show clear benefit from vitamin $\mathrm{C}$ due to the absence of positive effect on 43 out of 46 secondary outcomes. In COVID-19 patients, one RCT compared high dose vitamin C (24 gm I.V. daily) for a week versus placebo and showed no benefit in any of the outcome parameters (ventilator-free days, mortality, length of stay, and SOFA score) [65]. There are three large trials that are currently in progress.

Little consensus exists for the use of these micronutrients in patients with COVID-19 disease. The SCCM/ASPEN joint guidelines and Australian Society of Parenteral and Enteral Nutrition (AUSPEN) were not able to make formal recommendations based on the paucity of data in this population of patients $[1 \cdot, 66]$. The European Society for Clinical Nutrition and Metabolism (ESPEN) recommended providing the daily allowance of micronutrients only [67]. However, there is new consideration for use of vitamin D in COVID-19 patients who are hospitalized to attenuate the disease process enough to prevent transfer to the ICU because of results from the PO Calciferol trial [56]. The University of Louisville survey showed that vitamin $\mathrm{C}$ usage was reported in $42 \%, 45 \%$ reported vitamin D usage, $45 \%$ reported zinc usage, and melatonin usage was reported in $16 \%$ [7]. At this point, it may be reasonable to check levels of zinc, vitamin D, and selenium (and replete if necessary). Appropriate doses to consider in COVID-19 patients would be $20-40 \mathrm{mg}$ of elemental zinc, 3000-5000 IU of vitamin D daily or 50,000 IU weekly, and 250-500 $\mu$ g daily of selenium. Thiamine should be repleted empirically if there is suspicion for refeeding syndrome. No further recommendations for a COVID-19-specific antioxidant cocktail can be made until stronger data are available.

\section{Conclusions}

Bias, prejudices, and misconceptions carried over from traditional critical care impede effective nutritional support in this pandemic. Realistic fear of aspiration in patients with little or no pulmonary reserve, risk of bowel ischemia in conditions of hypercoagulation, and the real risk from the unpredictability of this disease process are all issues that need to be appropriately addressed. Identifying competent individuals for leadership, setting the tone for ICU management, and establishing protocols are all necessary to improve the delivery of nutritional therapy in critically ill patients with COVID-19 disease. 
Fig. 6 Choice of institutional strategies to provide optimal nutritional therapy in COVID19 disease: early initiation of small bowel feeding (a) (reproduced with permission from Chris Gralapp) versus gastric feeding with early supplementation of parenteral nutrition (b) (reproduced from Maria Palma - reference [68])
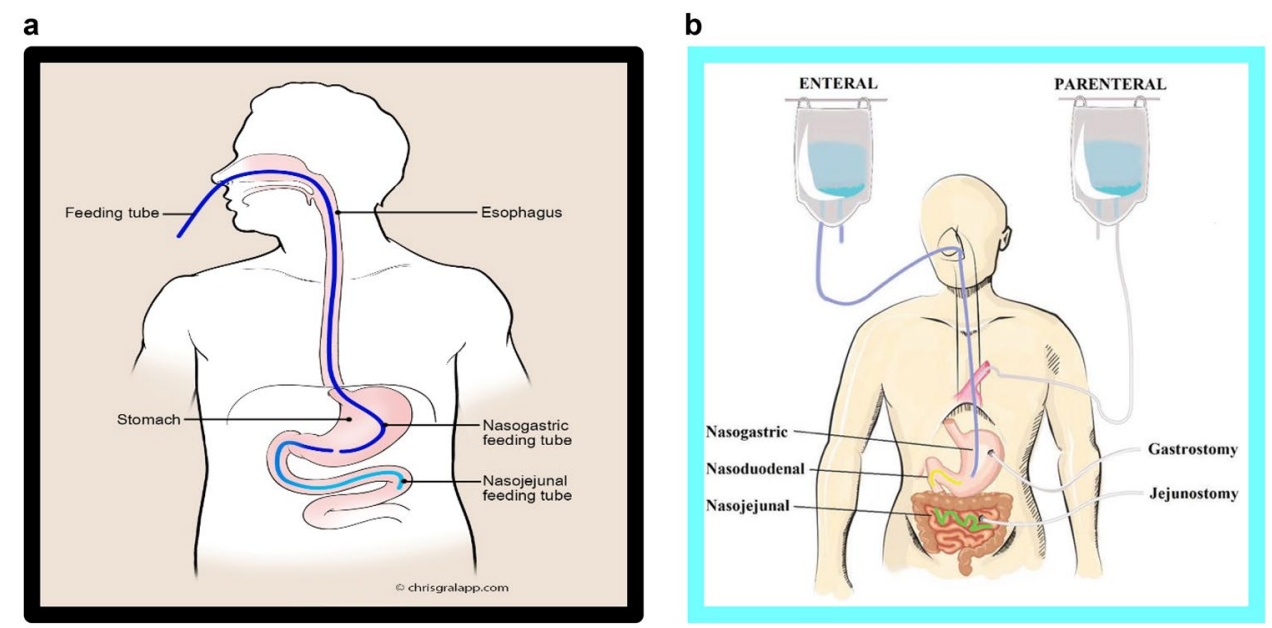

Medical centers should consider an institutional selfevaluation to determine the culture, leadership, and expertise of their ICU Setting. As an institution, a baseline strategy for better delivery of nutritional therapy comes down to two choices. One choice would be a base strategy of early initiation of small bowel feeding, where every COVID-19 patient undergoes placement of a post-pyloric feeding tube using a GPS-guided or optically guided enteral access device. Once access is secured, the small bowel feeding should be infused at the same rate whether the patient is in the prone or supine position (Fig. 6a). The other strategy would be to start oral diet or gastric feeding in all COVID-19 patients and consider adding supplemental PN within 2 days of admission to the ICU if requirements are not met (Fig. 6b) [68]. It is reasonable to use a low rate of infusion of EN with proning or while patients are on NIPPV per the intensivist, as long as the early supplemental PN can make up the difference. The University of Louisville Survey showed that the majority (58\%) of critically ill patients with COVID-19 disease got less than $60 \%$ of goal energy and protein requirements throughout hospitalization in the ICU [7]. The key issue is that prolonged attempts at gastric feeding alone lead to dramatic underfeeding and unacceptable deterioration of nutritional status.

There are noguarantees for the patient who survives COVID-19 that an optimal conditionexists at time of discharge. The goal should not be limited simply to sending thesepatients out of the ICU and hospital to their homes, as several studies have shownthat only $12.6 \%$ patients were symptom-free and $44.1 \%$ had worsened quality oflife after discharge $[69,70]$. Up to $67 \%$ of patients develop post-extubation dysphagia and EN is needed in addition to oralintake in $24 \%$ of patients after discharge [71] Up to20\% of patients develop Post-Traumatic Stress Disorder (PTSD) because of theirexperience in the ICU, and there is always a concern that patients might failto resolve the initial infection or rarely that they might get re-infected and haveto go back in to the hospital and the ICU [71].
In summary, COVID-19 is truly a unique disease process, with prolongation of the hypermetabolic hyperinflammatory phase of critical illness. The constraints of this disease create considerable barriers to nutritional therapy. Beliefs and prejudices regarding the need for aggressive EN and earlier use of supplemental PN must be re-addressed. This pandemic has raised new challenges, and institutions will need either re-acquire the skill set for sufficient delivery of EN or re-define the role of supplemental PN in critical illness.

\section{Declarations}

Human and Animal Rights and Informed Consent This article does not contain any studies with human or animal subjects performed by any of the authors.

\section{References}

\section{Papers of particular interest, published recently, have been highlighted as:}

\section{- Of importance}

1. Martindale R, Patel JJ, Taylor B, Arabi YM, Warren M, McClave SA. Nutrition therapy in critically ill patients with coronavirus disease 2019. JPEN J Parenter Enteral Nutr. 2020;44(7):1174-1184. This guidance was the first nutritional guidance from ASPEN for critically ill COVID-19 patients.

2. McClave SA, Taylor BE, Martindale RG, et al. Guidelines for the provision and assessment of nutrition support therapy in the adult critically ill patient: Society of Critical Care Medicine (SCCM) and American Society for Parenteral and Enteral Nutrition (A.S.P.E.N.). JPEN J Parenter Enteral Nutr. 2016;40(2):159-211. (This is the most recent guideline from ASPEN and SCCM on provision and assessment of nutrition 
in the critically ill patients. The COVID-19 guidance (reference 1) was mainly based on this guidline.)

3. Aboubakr HA, Sharafeldin TA, Goyal SM. Stability of SARS$\mathrm{CoV}-2$ and other coronaviruses in the environment and on common touch surfaces and the influence of climatic conditions: A review. Transbound Emerg Dis. 2021;68(2):296-312.

4. Galanopoulos M, Gkeros F, Doukatas A, et al. COVID-19 pandemic: pathophysiology and manifestations from the gastrointestinal tract. World J Gastroenterol. 2020;26(31):4579-88.

5. Petersen E, Koopmans M, Go U, et al. Comparing SARS-CoV-2 with SARS-CoV and influenza pandemics. Lancet Infect Dis. 2020;20(9):e238-44.

6. Wu Z, McGoogan JM. Characteristics of and important lessons from the coronavirus disease 2019 (COVID-19) outbreak in China: summary of a report of 72314 Cases From the Chinese Center for Disease Control and Prevention. JAMA. 2020;323(13):1239-42.

7. Richardson S, Hirsch JS, Narasimhan M, et al. Presenting characteristics, comorbidities, and outcomes among 5700 patients Hospitalized With COVID-19 in the New York City Area. JAMA. 2020;323(20):2052-9.

8. Mechanick JI, Carbone S, Dickerson RN, et al. Clinical nutrition research and the COVID-19 pandemic: a scoping review of the ASPEN COVID-19 Task Force on nutrition research. JPEN J Parenter Enteral Nutr. 2021;45(1):13-31 This review article from ASPEN COVID-19 Task Force elaborated multiple critical areas for urgent nutrition research to improve nutrition care during the COVID-19 pandemic.

9. Suliman S, SA McClave, BE Taylor, et al. Barriers to nutritional therapy in the critically ill patient with COVID-19 disease. Submitted to JPEN J Parenter Enteral Nutr. 2021. This article shows the survey we conducted at the University of Louisville to identify barriers to nutritional therapy in critically ill patinets with COVID-19.

10. Horwitz LI, Jones SA, Cerfolio RJ, et al. Trends in COVID-19 risk-adjusted mortality rates. J Hosp Med. 2021;16(2):90-2.

11. Caramelo F, Ferreira N, Oliveiros B. Estimation of risk factors for COVID-19 mortality - preliminary results. medRxiv. 2020:2020.2002.2024.20027268.

12. Cena H, Chieppa M. Coronavirus disease (COVID-19-SARS$\mathrm{CoV}-2)$ and nutrition: is infection in Italy suggesting a connection? Front Immunol. 2020;11(944).

13. Belanger MJ, Hill MA, Angelidi AM, Dalamaga M, Sowers JR, Mantzoros CS. Covid-19 and disparities in nutrition and obesity. N Engl J Med. 2020;383(11):e69.

14. Davenport DL, Xenos ES, Hosokawa P, Radford J, Henderson WG, Endean ED. The influence of body mass index obesity status on vascular surgery 30 -day morbidity and mortality. J Vasc Surg. 2009;49(1):140-147, 147.e141; discussion 147.

15. Finer N, Garnett SP, Bruun JM. COVID-19 and obesity. Clin Obes. 2020;10(3):e12365.

16. Lui B, Samuels JD, White RS. Potential pathophysiology of COVID-19 in patients with obesity. Comment on $\mathrm{Br}$ J Anaesth 2020; 125: e262-e263. Br J Anaesth. 2020;125(3):e283-e284.

17. Cave MC, Hurt RT, Frazier TH, et al. Obesity, inflammation, and the potential application of pharmaconutrition. Nutr Clin Pract. 2008;23(1):16-34.

18. Whittle J, Molinger J, MacLeod D, Haines K, Wischmeyer PE. Persistent hypermetabolism and longitudinal energy expenditure in critically ill patients with COVID-19. Crit Care. 2020;24(1):581 This prospective observational study highlighted the unique metabolic response in patients with COVID-19 using indirect calometry.

19. Carson JS, Khosrozadeh H, Norbury WB, Herndon DN. 28 Nutritional needs and support for the burned patient. In: Herndon
DN, editor. Total burn care (Fifth Edition). Elsevier; 2018. p. 287- 300.e282.

20. Mathias B, Szpila BE, Moore FA, Efron PA, Moldawer LL. A review of GM-CSF therapy in sepsis. Medicine (Baltimore). 2015;94(50):e2044.

21. Kovacevic MP, Dube KM, Lupi KE, Szumita PM, DeGrado JR. Evaluation of hypertriglyceridemia in critically ill patients with coronavirus disease 2019 receiving propofol. Crit Care Explor. 2021;3(1):e0330.

22. Lippi G, South AM, Henry BM. Electrolyte imbalances in patients with severe coronavirus disease 2019 (COVID-19). Ann Clin Biochem. 2020;57(3):262-5.

23. Kallet RH. A comprehensive review of prone position in ARDS. Respir Care. 2015;60(11):1660-87.

24. Behrens S, Kozeniecki M, Knapp N, Martindale RG. Nutrition support during prone positioning: an old technique reawakened by COVID-19. Nutr Clin Pract. 2021;36(1):105-9.

25. Reignier J, Dimet J, Martin-Lefevre L, et al. Before-after study of a standardized ICU protocol for early enteral feeding in patients turned in the prone position. Clin Nutr. 2010;29(2):210-6.

26. Sams VG, Lawson CM, Humphrey CL, et al. Effect of rotational therapy on aspiration risk of enteral feeds. Nutr Clin Pract. 2012;27(6):808-11.

27. Reignier J, Thenoz-Jost N, Fiancette M, et al. Early enteral nutrition in mechanically ventilated patients in the prone position. Crit Care Med. 2004;32(1):94-9.

28. Ohbe H, Jo T, Yamana H, Matsui H, Fushimi K, Yasunaga H. Early enteral nutrition for cardiogenic or obstructive shock requiring venoarterial extracorporeal membrane oxygenation: a nationwide inpatient database study. Intensive Care Med. 2018;44(8):1258-65.

29. Park J, Heo E, Song IA, et al. Nutritional support and clinical outcomes in critically ill patients supported with veno-arterial extracorporeal membrane oxygenation. Clin Nutr. 2020;39(8):2617-23.

30. Ohbe H, Jo T, Matsui H, Fushimi K, Yasunaga H. Early enteral nutrition in patients undergoing sustained neuromuscular blockade: a propensity-matched analysis using a nationwide inpatient database. Crit Care Med. 2019;47(8):1072-80.

31. Marik PE, Pinsky M. Death by parenteral nutrition. Intensive Care Med. 2003;29(6):867-9.

32. Rice TW, Wheeler AP, Thompson BT, et al. Initial trophic vs full enteral feeding in patients with acute lung injury: the EDEN randomized trial. JAMA. 2012;307(8):795-803.

33. Casaer MP, Mesotten D, Hermans G, et al. Early versus late parenteral nutrition in critically ill adults. $\mathrm{N}$ Engl $\mathrm{J}$ Med. 2011;365(6):506-17.

34. Heidegger CP, Berger MM, Graf S, et al. Optimisation of energy provision with supplemental parenteral nutrition in critically ill patients: a randomised controlled clinical trial. Lancet. 2013;381(9864):385-93.

35. Allingstrup MJ, Kondrup J, Wiis J, et al. Early goal-directed nutrition versus standard of care in adult intensive care patients: the single-centre, randomised, outcome assessor-blinded EAT-ICU trial. Intensive Care Med. 2017;43(11):1637-47.

36. Singer P, Anbar R, Cohen J, et al. The tight calorie control study (TICACOS): a prospective, randomized, controlled pilot study of nutritional support in critically ill patients. Intensive Care Med. 2011;37(4):601-9.

37. Singer P, De Waele E, Sanchez C, et al. TICACOS international: a multi-center, randomized, prospective controlled study comparing tight calorie control versus Liberal calorie administration study. Clin Nutr. 2021;40(2):380-7.

38. Wischmeyer PE, Hasselmann M, Kummerlen C, et al. A randomized trial of supplemental parenteral nutrition in underweight and overweight critically ill patients: the TOP-UP pilot trial. Crit Care. 2017;21(1):142. 
39. Berger MM. Can oxidative damage be treated nutritionally? Clin Nutr. 2005;24(2):172-83.

40. D Heyland et al. Critical care nutrition systematic reviews. Parenteral Selenium supplementation in critically ill patients. March 2021.

41. Zhang J, Taylor EW, Bennett K, Saad R, Rayman MP. Association between regional selenium status and reported outcome of COVID-19 cases in China. Am J Clin Nutr. 2020;111(6):1297-9.

42. Moghaddam A, Heller RA, Sun Q, et al. Selenium deficiency is associated with mortality risk from COVID-19. Nutrients. 2020;12(7).

43. Heyland DK, Jones N, Cvijanovich NZ, Wong H. Zinc supplementation in critically ill patients: a key pharmaconutrient? JPEN J Parenter Enteral Nutr. 2008;32(5):509-19.

44. Frontera JA, Rahimian JO, Yaghi S, et al. Treatment with zinc is associated with reduced in-hospital mortality among COVID-19 patients: a multi-center cohort study. Res Sq. 2020.

45. Thomas S, Patel D, Bittel B, et al. Effect of high-dose zinc and ascorbic acid supplementation vs usual care on symptom length and reduction among ambulatory patients with SARS-CoV-2 infection: the COVID A to $\mathrm{Z}$ randomized clinical trial. JAMA Netw Open. 2021;4(2):e210369.

46. Putzu A, Belletti A, Cassina T, et al. Vitamin D and outcomes in adult critically ill patients. A systematic review and meta-analysis of randomized trials. J Crit Care. 2017;38:109-114.

47. Weng H, Li JG, Mao Z, Zeng XT. Randomised trials of vitamin $\mathrm{D}(3)$ for critically ill patients in adults: systematic review and meta-analysis with trial sequential analysis. Intensive Care Med. 2017;43(2):277-8.

48. Ginde AA, Brower RG, Caterino JM, et al. Early high-dose vita$\min \mathrm{D}(3)$ for critically ill, vitamin D-deficient patients. N Engl J Med. 2019;381(26):2529-40.

49. D'Avolio A, Avataneo V, Manca A, et al. 25-Hydroxyvitamin D concentrations are lower in patients with positive PCR for SARSCoV-2. Nutrients. 2020;12(5).

50. Hastie CE, Mackay DF, Ho F, et al. Vitamin D concentrations and COVID-19 infection in UK Biobank. Diabetes Metab Syndr. 2020;14(4):561-5.

51. Meltzer DO, Best TJ, Zhang H, Vokes T, Arora V, Solway J. Association of vitamin D status and other clinical characteristics with COVID-19 test results. JAMA Netw Open. 2020;3(9):e2019722.

52. Merzon E, Tworowski D, Gorohovski A, et al. Low plasma $25(\mathrm{OH})$ vitamin $\mathrm{D}$ level is associated with increased risk of COVID-19 infection: an Israeli population-based study. Febs j. 2020;287(17):3693-702.

53. Panagiotou G, Tee SA, Ihsan $\mathrm{Y}$, et al. Low serum 25-hydroxyvitamin D $(25[\mathrm{OH}] \mathrm{D})$ levels in patients hospitalized with COVID19 are associated with greater disease severity. Clin Endocrinol (Oxf). 2020;93(4):508-11.

54. Z Raisi-Estabragh AR Martineau EM Curtis D Vitamin coronavirus disease, et al 2019 (COVID-19): rapid evidence review Aging Clin Exp Res 2021111

55. Xu Y, Baylink DJ, Chen CS, et al. The importance of vitamin $\mathrm{d}$ metabolism as a potential prophylactic, immunoregulatory and neuroprotective treatment for COVID-19. J Transl Med. 2020;18(1):322

56. Entrenas Castillo M, Entrenas Costa LM, Vaquero Barrios JM, et al. Effect of calcifediol treatment and best available therapy versus best available therapy on intensive care unit admission and mortality among patients hospitalized for COVID-19: A pilot randomized clinical study. J Steroid Biochem Mol Biol. 2020;203:105751.

57. Chang P, Liao Y, Guan J, et al. Combined treatment with hydrocortisone, vitamin $\mathrm{C}$, and thiamine for sepsis and septic shock: a randomized controlled trial. Chest. 2020;158(1):174-82.

58. Fujii T, Luethi N, Young PJ, et al. Effect of vitamin C, hydrocortisone, and thiamine vs hydrocortisone alone on time alive and free of vasopressor support among patients with septic shock: the VITAMINS randomized clinical trial. JAMA. 2020;323(5):423-31.

59. Hwang SY, Ryoo SM, Park JE, et al. Combination therapy of vitamin $\mathrm{C}$ and thiamine for septic shock: a multi-centre, double-blinded randomized, controlled study. Intensive Care Med. 2020;46(11):2015-25.

60. Iglesias J, Vassallo AV, Patel VV, Sullivan JB, Cavanaugh J, Elbaga Y. Outcomes of metabolic resuscitation using ascorbic acid, thiamine, and glucocorticoids in the early treatment of sepsis: the ORANGES trial. Chest. 2020;158(1):164-73.

61. Moskowitz A, Huang DT, Hou PC, et al. Effect of ascorbic acid, corticosteroids, and thiamine on organ injury in septic shock: the ACTS randomized clinical trial. JAMA. 2020;324(7):642-50.

62. Sevransky JE, Rothman RE, Hager DN, et al. Effect of vitamin $\mathrm{C}$, thiamine, and hydrocortisone on ventilator- and vasopressorfree days in patients with sepsis: the VICTAS randomized clinical trial. JAMA. 2021;325(8):742-50.

63. Wani SJ, Mufti SA, Jan RA, et al. Combination of vitamin C, thiamine and hydrocortisone added to standard treatment in the management of sepsis: results from an open label randomised controlled clinical trial and a review of the literature. Infect Dis (Lond). 2020;52(4):271-8.

64. Fowler AA 3rd, Truwit JD, Hite RD, et al. Effect of vitamin C infusion on organ failure and biomarkers of inflammation and vascular injury in patients with sepsis and severe acute respiratory failure: the CITRIS-ALI randomized clinical trial. JAMA. 2019;322(13):1261-70.

65. Zhang J, Rao X, Li Y, et al. Pilot trial of high-dose vitamin $\mathrm{C}$ in critically ill COVID-19 patients. Ann Intensive Care. 2021;11(1):5.

66. Warrillow S, Austin D, Cheung W, et al. ANZICS guiding principles for complex decision making during the COVID-19 pandemic. Crit Care Resusc. 2020;22(2):98-102.

67. Barazzoni R, Bischoff SC, Breda J, et al. ESPEN expert statements and practical guidance for nutritional management of individuals with SARS-CoV-2 infection. Clin Nutr. 2020;39(6):1631-8.

68. Palma MA, Mahía J, Simón MJ, Molina F, Puerto A. Enteral feeding: brain-visceral interactions in the processing of nutrients. 2019.

69. Post-COVID-19 global health strategies: the need for an interdisciplinary approach. Aging Clin Exp Res. 2020;32(8):1613-1620.

70. Carfî A, Bernabei R, Landi F. Persistent symptoms in patients after acute COVID-19. JAMA. 2020;324(6):603-5.

71. Thibault R, Seguin P, Tamion F, Pichard C, Singer P. Nutrition of the COVID-19 patient in the intensive care unit (ICU): a practical guidance. Crit Care. 2020;24(1):447.

Publisher's Note Springer Nature remains neutral with regard to jurisdictional claims in published maps and institutional affiliations. 